\section{Adequação da demanda de crianças e adolescentes atendidos na Unidade de Emergência em Maceió, Alagoas, Brasil}

\section{Meeting the demand for services of children and adolescents at the Emergency Unit in Maceió, in the Brazilian State of Alagoas}

Dione Alencar Simons 1

Isabella Lopes Monlleó 2

Sofia Alencar Simons 3

José Luiz Araújo Júnior 4
1-3 Núcleo de Saúde da Criança e do Adolescente. Faculdade de Medicina. Universidade Estadual de Ciências da Saúde de Alagoas - UNCISAL. Rua Dr. Jorge de Lima, 113. Trapiche da Barra. Maceió, AL, Brasil. CEP: 57.010-300.

E-mail: dione_simons@yahoo.com.br

4 Departamento de Saúde Coletiva. Centro de Pesquisas Aggeu

Magalhães. Fundação Oswaldo Cruz. Recife, PE, Brasil.

\section{Abstract}

Objective: to evaluate the extent to which the demand for services from children and adolescents are met by the Doutor Armando Lages Emergency Unit in Maceio, in the Brazilian State of Alagoas.

Methods: an observational cross-section study was carried out of 2,153 medical records of children and adolescents attending the Emergency unit in 1998, 2001 and 2004. The variables were sex, age, sector and time attended, place of residence, hospitalization, type of injury and extent to which the demand was met, according to the criterion established by Furtado et al. The chi-squared test was used to investigate associations between the variables under study, with a confidence level of $95 \%$.

Results: of the 2,153 medical records analyzed, 1792 (83.2\%) were classified as inadequate. Most patients were male $(1252-58.2 \%)$, aged between 10 and 18 years $(931-43.2 \%)$, resident in Maceió $(1742$ $-81.1 \%)$, attended in the afternoon (800-37.2\%), at the Pediatrics Centre with injuries covered by Chapter XIX of the International Classification of Diseases (1039-48.3\%). There were more inadequate medical records for females aged between 10 and 18 years, attended in the morning, residing in close proximity to the Emergency Unit.

Conclusions: there was a high percentage of inadequate service provision for children and adolescents at the Emergency Unit. This result suggests the health needs of this group are not being met by the basic National Health System in the State of Alagoas. Key words Child, Adolescent, Morbidity, Emergency medicine

\section{Resumo}

Objetivos: avaliar a adequação da demanda de crianças e adolescentes atendidos na Unidade de Emergência Doutor Armando Lages em Maceió Alagoas, Brasil.

Métodos: estudo observacional-transversal de 2153 registros de crianças e adolescentes atendidos na Unidade de Emergência, em 1998, 2001 e 2004. Variáveis: sexo, idade, setor e horário do atendimento, procedência, internação, tipo de agravo e adequação da demanda, segundo critério de Furtado et al. Para testar associações entre variáveis de interesse foi utilizado teste do Qui-quadrado, adotandose nivel de confiança de $95 \%$.

Resultados: entre os 2153 registros analisados, 1792 (83,2\%) foram classificados como não adequados. Predominaram usuários do sexo masculino (1252 - 58,2\%), entre 10 e 18 anos (93143,2\%), residentes em Maceió $(1742-81,1 \%)$, atendidos à tarde $(800-37,2 \%)$, no setor de pediatria e com agravos do Capítulo XIX da Classificação Internacional de Doenças (1039 - 48,3\%). A não adequação da demanda foi maior no sexo feminino, entre 10 e 18 anos, atendida no horário da manhã e residente próximo à Unidade de Emergência.

Conclusões: verificou-se alto percentual de não adequação da demanda de crianças e adolescentes à Unidade de Emergência. Esse resultado sinaliza para o não atendimento às necessidades de saúde na rede básica do Sistema Único de Saúde em Alagoas.

Palavras-chave Criança, Adolescente, Morbidade, Medicina de Emergência 


\section{Introdução}

A despeito dos avanços do Sistema Único de Saúde (SUS), ainda são citadas dificuldades quanto à igualdade de acesso da população às ações e serviços de saúde e à qualidade da atenção. ${ }^{1}$ Uma das áreas que expressa bem esses problemas é a dos serviços de urgência/emergência hospitalar. Esses serviços apresentam escassez de recursos humanos e materiais e inadequação entre oferta e demanda, devido à absorção de uma grande quantidade de casos não emergenciais que poderiam ser atendidos em serviços da atenção básica ou especializada, ou em serviços de urgência de menor complexidade. ${ }^{1}$

A demanda excessiva compromete a qualidade da assistência prestada, tanto para os que realmente necessitam de atendimento em um serviço com melhor capacidade tecnológica, como para aqueles que procuram o serviço com situações de saúde que, na sua maioria, não exigem atendimento médico emergencial. ${ }^{2}$ Esse fato atinge todas as faixas de idade e ocorre no Brasil,3 em outros países em desenvolvimento, como Chile 4 e México, 5 e também em países desenvolvidos, cuja realidade social, econômica e cultural é distinta, a exemplo da Espanha, ${ }^{6}$ França, 7 Canadá8 e Estados Unidos. 9

Especificamente na faixa etária de crianças e adolescentes, estudo realizado em cinco serviços públicos de urgência/emergência da cidade do Recife revela que $63,5 \%$ dos usuários apresentavam problemas de saúde que poderiam ser solucionados na rede básica. ${ }^{2}$

Percentuais de não adequação variam entre diferentes estudos a depender do critério empregado.10-12 Essa variação se deve à inexistência de consenso em torno da definição de urgência, não urgência e emergência, assim como há controvérsias quanto à definição de qual nível assistencial é mais adequado para cada tipo de situação. Além disso, a percepção do que é urgente difere entre as pessoas que buscam os serviços de urgência hospitalar e entre os profissionais de saúde que as atendem.

Segundo o Conselho Federal de Medicina, ${ }^{13}$ urgência "é a ocorrência imprevista de agravo à saúde com ou sem risco potencial de vida, cujo portador necessita de assistência médica imediata". Emergência "é a constatação médica de condições de agravo à saúde que impliquem em risco iminente de vida ou sofrimento intenso, exigindo, portanto, tratamento médico imediato".

Paim 14 conceitua urgência, não urgência e emergência de acordo com o risco de morte. No caso de um atendimento de emergência, o risco de morte é iminente, no caso de uma urgência, o risco existe, mas não é iminente e, no caso dos problemas 'de rotina', esse risco é inexistente.

Com base nas definições de urgência e emergência, foram elaborados protocolos de avaliação da demanda, sendo definidos como casos não adequados aqueles não urgentes/emergentes, passíveis de resolução na atenção primária. $2,10,12,15-16$

Em nosso meio, Furtado et al. ${ }^{3}$ elaboraram, testaram e validaram um protocolo para avaliar casos apropriados para o atendimento em serviço de emergência de média e alta complexidade. Esse protocolo é constituído de uma lista de agravos de saúde, elencados por especialistas em emergência e trauma de um hospital geral, público e estadual, com nível de complexidade III no SUS, cuja missão institucional é o atendimento de emergência, por meio de consultas ambulatoriais e internação.

O objetivo desta pesquisa é avaliar a adequação da demanda de crianças e adolescentes atendidos na Unidade de Emergência (UE), em Maceió, Alagoas, por meio da aplicação do protocolo de Furtado et al. 3 A utilização desse protocolo se deveu às características semelhantes entre este serviço e aquele que serviu de base para sua elaboração.

\section{Métodos}

Trata-se de um estudo com desenho observacionaltransversal, utilizando registros médicos (fichas de atendimento ambulatorial e prontuários) dos usuários da Unidade de Emergência, em Maceió, Alagoas, durante o período de 1 de janeiro a 31 de dezembro dos anos de 1998, 2001 e 2004. A escolha do ano de 1998 considerou o início do processo de municipalização e do Programa Saúde da Família (PSF) no Estado de Alagoas. Para análise das possíveis mudanças no perfil de morbidade foi adotado critério de observação trienal, sendo utilizados os anos de 2001 e 2004.

Os dados foram coletados no Setor de Arquivo Médico, tendo-se obtido as informações referentes aos seguintes campos: número do registro; data, hora e setor do atendimento; idade; sexo; procedência, diagnóstico principal e tipo de assistência.

O tamanho amostral foi estabelecido a partir dos resultados do projeto piloto realizado com 3000 registros, referentes aos atendimentos nos anos de 1998 e 2004. Foram identificados os seis grupos de causas (capítulos) da Classificação Internacional de Doenças (CID 10) cujos quantitativos foram maiores do que 100 casos em pelo menos um dos anos estudados. O grupo com menor proporção de casos $(6,53 \%)$ ocorreu no capítulo IX - Doenças do 
Aparelho Circulatório - e a diferença entre os dois anos $(1998-8,73 \%$ e $2004-6,53 \%)$ foi de $2,2 \%$. Com esse resultado, foi possível calcular um tamanho mínimo da amostra em 2368 registros por ano.

A seleção dos registros se deu de forma sistemática. O total de atendimentos realizados em cada ano da pesquisa foi dividido pelo número amostral e a partir deste se encontrou o intervalo da seleção: em 1998, um a cada 61 registros (144982 atendimentos $/ 2368=61)$; em 2001 e 2004, um a cada 67 registros (159189 atendimentos/2368 =67; 159793 atendimentos $/ 2368=67)$. Com base nesses cálculos, foram sorteadas, entre os números 1 e 61 e entre 1 e 67, os registros para início da coleta dos dados nos respectivos anos.

Foram excluídos os registros com letra ilegível, sem preenchimento dos campos selecionados e de vítimas que chegaram sem vida à unidade, por não constar o diagnóstico. Os documentos excluídos foram substituídos pelo seguinte à numeração.

A amostra final totalizou 7104 registros. Para este estudo foram selecionados apenas os atendimentos referentes aos usuários entre 0 e 18 anos de idade, o que correspondeu a 2153 registros válidos para análise.

Foi estudada, como variável dependente, a adequação da demanda de acordo com protocolo de Furtado et al.,3 e, como variáveis independentes, sexo, idade, setor (clínicas cirúrgicas ou não cirúrgicas), horário do atendimento, procedência, tipo de assistência (ambulatorial ou internação), e o tipo de agravo conforme a CID.

Os casos foram classificados segundo o protocolo de Furtado et al.,3 que estabelece como demanda adequada os seguintes agravos, independente se o atendimento resultou em internação ou não: abdome agudo, abrasão de córnea, acidente ofídico, acidentes por escorpião, afogamento, amputação traumática, aneurisma de aorta abdominal, acidente vascular cerebral, acidente vascular cerebral hemorrágico, acidente vascular cerebral isquêmico, avulsão de dentes traumático, celulite peri orbitária, ceratite fotoelétrica, contusão torácica, corpo estranho em orofaringe, corpo estranho no olho, crise convulsiva, epilepsia, evisceração de olho, fratura exposta, hematoma ocular, hematoma subdural crônico, hemorragia digestiva alta ou baixa, hemorragia ocular, hemorragia subaracnóidea, hifema traumático, intoxicação exógena, isquemia crítica, laceração de córnea, lesões penetrantes, linfangite necrotizante, pneumotórax, politraumatismos, queimaduras, trauma de crânio, trauma de face, trauma ocular, trauma raquimedular, tumor cerebral.

A idade foi agrupada por faixas etárias: menor de um ano; de 1 a 4 anos; de 5 a 9 anos; e de 10 a 18 anos. Os setores de atendimento foram agrupados em clínicas cirúrgicas (cirurgia geral e cirurgia bucomaxilo-facial) e clínicas não cirúrgicas (clínica médica, pediatria, queimados, ortopedia/traumatologia, otorrinolaringologia, oftalmologia, odontologia). O horário do atendimento foi agrupado por períodos: madrugada (00h00 às 5h59), manhã (06h00 às $11 \mathrm{~h} 59)$, tarde (12h00 às $17 \mathrm{~h} 59)$ e noite (18h00 às 23h59). O local de procedência do paciente foi categorizado por microrregiões de saúde do Estado de Alagoas e por distritos sanitários da cidade de Maceió (Figura 1). O tipo de assistência foi agrupado em ambulatorial (usuários liberados para o domicílio após o atendimento) e internação.

Para construir o perfil de morbidade, os agravos foram codificados e classificados segundo a lista tabular de inclusões e subcategorias de quatro caracteres da Classificação Internacional de Doenças $10^{\mathrm{a}}$ revisão (CID 10).

Realizou-se a análise descritiva dos atendimentos segundo as características estudadas. A associação entre adequação da demanda e as variáveis de interesse foi testada utilizando o qui-quadrado. Adotou-se nível de significância de 0,05. Os dados obtidos foram analisados por meio do programa Epi Info 2007, versão Windows 3.43.

Este estudo foi aprovado pelo Comitê de Ética em Pesquisa da Universidade Estadual de Ciências da Saúde de Alagoas, em 28 de fevereiro de 2005, protocolo $\mathrm{n}^{\mathrm{o}} 283$.

\section{Resultados}

O perfil e a adequação da demanda de crianças e adolescentes atendidos na UE estão apresentados na Tabela 1. Do total de atendimentos, 1792 (83,2\%) foram considerados não adequados a um serviço de urgência/emergência hospitalar de média e alta complexidade (nível III). As diferenças nos valores de não adequação da demanda nos anos da pesquisa não foram estatisticamente significativas $(p=0,827)$.

Quanto ao sexo, a maior proporção foi de crianças e adolescentes do sexo masculino (1252 $-58,2 \%$ ), porém os atendimentos de usuários do sexo feminino mostraram maior 'não adequação'(769 $85,3 \%$ ). Houve diferenças estatisticamente significativas entre o total de casos atendidos por sexo em relação à adequação da demanda $(p=0,043)$.

Com relação à faixa etária, a maior proporção de atendimentos recaiu sobre o grupo de adolescentes, compreendido entre 10 e 18 anos, seguido pelo 


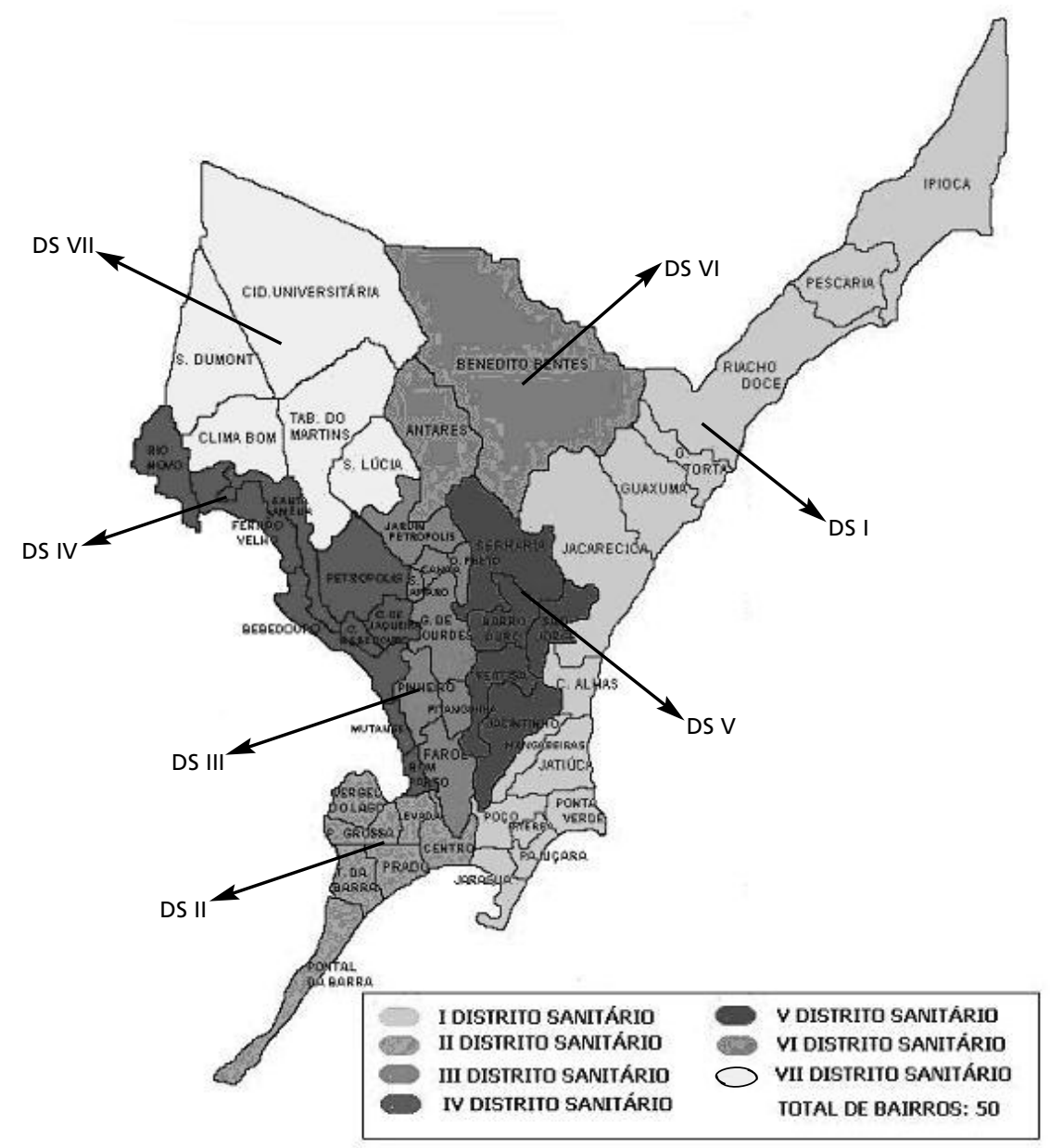

Fonte: Secretaria Municipal de Saúde de Maceió-AL.

grupo de crianças de 1 a 4 anos, conforme se observa na Tabela 1. Foram encontrados elevados valores de não adequação da demanda em todas as faixas etárias, tendo sido um pouco mais elevado no grupo de 10 a 18 anos (796-86,5\%). Houve diferenças estatisticamente significativas entre o total de casos atendidos por faixa etária em relação à adequação da demanda $(p=0,047)$.

Houve maior proporção de atendimentos no período da tarde (800-37,2\%), seguido pelo período da noite (685-31,8\%), manhã (555-25,8\%) e madrugada $(113-5,2 \%)$. O período da manhã foi o que apresentou maior não adequação da demanda (491-88,5\%), conforme se observa na Tabela 1 . Houve diferenças estatisticamente significativas entre o total de casos atendidos por período em relação à adequação da demanda $(p<0.001)$.

Em relação à procedência, a grande maioria das crianças e adolescentes foi composta por residentes em Maceió (1742-81,1\%) e por aqueles residentes em municípios mais próximos à capital, que compõem a primeira microrregião de saúde de Alagoas (Tabela 2).

Entre os residentes em Maceió, a maior proporção de atendimentos foi de crianças e adolescentes residentes no II distrito de saúde (784-45,0\%), onde se situa a UE, para o qual se verificou a maior não adequação da demanda (691-88,1\%) (Tabela 2).

A distribuição dos atendimentos em relação aos setores mostra a clínica pediátrica como a de maior aporte $(927-43,1 \%)$, de acordo com a Tabela 3. 
Agrupando as clínicas entre cirúrgicas e não cirúrgicas, observa-se que não houve diferença estatisticamente significativa entre o total de casos por grupo de clínicas (cirúrgicas e não cirúrgicas) em relação à adequação da demanda $(p=0,130)$.

A Tabela 4 apresenta o perfil de morbidade da UE segundo a Classificação Internacional de Doenças $10^{\mathrm{a}}$ Revisão, em relação à adequação da demanda. Os agravos mais frequentes compõem o capítulo XIX da CID 10: "Lesões, envenenamentos e algumas consequências de causas externas" (1039-48,3\%).Verificou-se que a totalidade de atendimentos cujos agravos compõem os capítulos I, III, IV, V, VIII, IX, X, XIII, XIV, XVI, XXI da CID
10 foram não adequados. A maior adequação ocorreu para os agravos do capítulo XX da CID 10 (21$32,8 \%)$.

Dos registros, $2043(94,9 \%)$ corresponderam a fichas de atendimento ambulatorial (usuários não internados) e $110(5,1 \%)$, a prontuários médicos (usuários internados). A não adequação da demanda entre os que não permaneceram e os que permaneceram internados foi, respectivamente, 1741 $(85,2 \%)$ e $51(46,4 \%)$. Houve diferenças estatisticamente significativas entre o total de casos atendidos por tipo de assistência em relação à adequação da demanda $(p<0.001)$.

Tabela 1

Distribuição dos atendimentos de crianças e adolescentes, segundo adequação da demanda, ano do atendimento, sexo, faixa etária e período do atendimento. Unidade de Emergência, Maceió-AL, 1998, 2001 e 2004.

\begin{tabular}{|c|c|c|c|}
\hline Adequação da demanda & Sim & Não & Total \\
\hline \multicolumn{4}{|l|}{ Ano* } \\
\hline 1998 & 120 & 625 & 745 \\
\hline 2001 & 123 & 602 & 725 \\
\hline 2004 & 118 & 565 & 863 \\
\hline \multicolumn{4}{|l|}{ Sexo\# } \\
\hline Masculino & 229 & 1023 & 1252 \\
\hline Feminino & 132 & 769 & 901 \\
\hline \multicolumn{4}{|l|}{ Faixa etária em anos§ } \\
\hline$<1$ & 24 & 124 & 148 \\
\hline $1-4$ & 124 & 499 & 623 \\
\hline $5-9$ & 78 & 373 & 451 \\
\hline $10-18$ & 135 & 796 & 931 \\
\hline \multicolumn{4}{|l|}{ Período do atendimento $€$} \\
\hline Madrugada & 23 & 90 & 113 \\
\hline Manhã & 64 & 491 & 555 \\
\hline Tarde & 161 & 639 & 800 \\
\hline Noite & 113 & 572 & 685 \\
\hline
\end{tabular}

${ }^{*} \chi^{2}=0.38 ; p=0,827 ; \#: \chi^{2}=4,71 ; p=0,029 ; \S \chi^{2}=7.94 ; p=0,047 ; € \chi^{2}=18.44 ; p<0,001$. 
Tabela 2

Distribuição dos atendimentos de crianças e adolescentes, segundo procedência por microrregiões de saúde de Alagoas e distritos sanitários de Maceió e adequação da demanda. Unidade de Emergência, Maceió-AL, 1998,2001 e 2004.

\begin{tabular}{|c|c|c|c|}
\hline Adequação da demanda & Sim & Não & Total \\
\hline 1 & 305 & 1633 & 1938 \\
\hline 2 & 6 & 27 & 33 \\
\hline 5 & 5 & 6 & 11 \\
\hline 6 & 0 & 8 & 8 \\
\hline 7 & 1 & 3 & 4 \\
\hline 8 & 3 & 7 & 10 \\
\hline 12 & 6 & 15 & 21 \\
\hline 13 & 5 & 13 & 18 \\
\hline \multicolumn{4}{|l|}{ Distritos sanitários** } \\
\hline I & 29 & 127 & 156 \\
\hline II & 93 & 691 & 784 \\
\hline III & 12 & 82 & 94 \\
\hline IV & 45 & 160 & 205 \\
\hline $\mathrm{V}$ & 38 & 160 & 198 \\
\hline VI & 11 & 62 & 73 \\
\hline
\end{tabular}

Fonte: *Plano Diretor de Regionalização das Ações de Saúde do Estado de Alagoas. 2002. Secretaria Estadual de Saúde; ** Secretaria Municipal de Saúde de Maceió.

Tabela 3

Distribuição dos atendimentos de crianças e adolescentes, segundo setor de atendimento e adequação da demanda. Unidade de Emergência, Maceió-AL, 1998, 2001 e 2004.

\begin{tabular}{|c|c|c|c|c|c|}
\hline Setor & $\begin{array}{c}\text { Total de } \\
\text { atendimentos }\end{array}$ & \multicolumn{2}{|c|}{ Adequados } & \multicolumn{2}{|c|}{ Não adequados } \\
\hline Pediatria & 927 & 125 & 13,5 & 802 & 86,5 \\
\hline Clínica Médica & 352 & 44 & 12,5 & 308 & 87,5 \\
\hline Clínica Cirúrgica & 166 & 32 & 19,3 & 134 & 80,7 \\
\hline Traumatologia & 119 & 64 & 53,8 & 55 & 46,2 \\
\hline Queimados & 54 & 54 & 100,0 & 00 & 0,0 \\
\hline Odontologia & 39 & 08 & 20,5 & 31 & 79,5 \\
\hline Cirurgia Buco-maxilo-facial & 09 & 05 & 55,6 & 04 & 44,4 \\
\hline Total & 2153 & 361 & 16,8 & 1792 & 83,2 \\
\hline
\end{tabular}




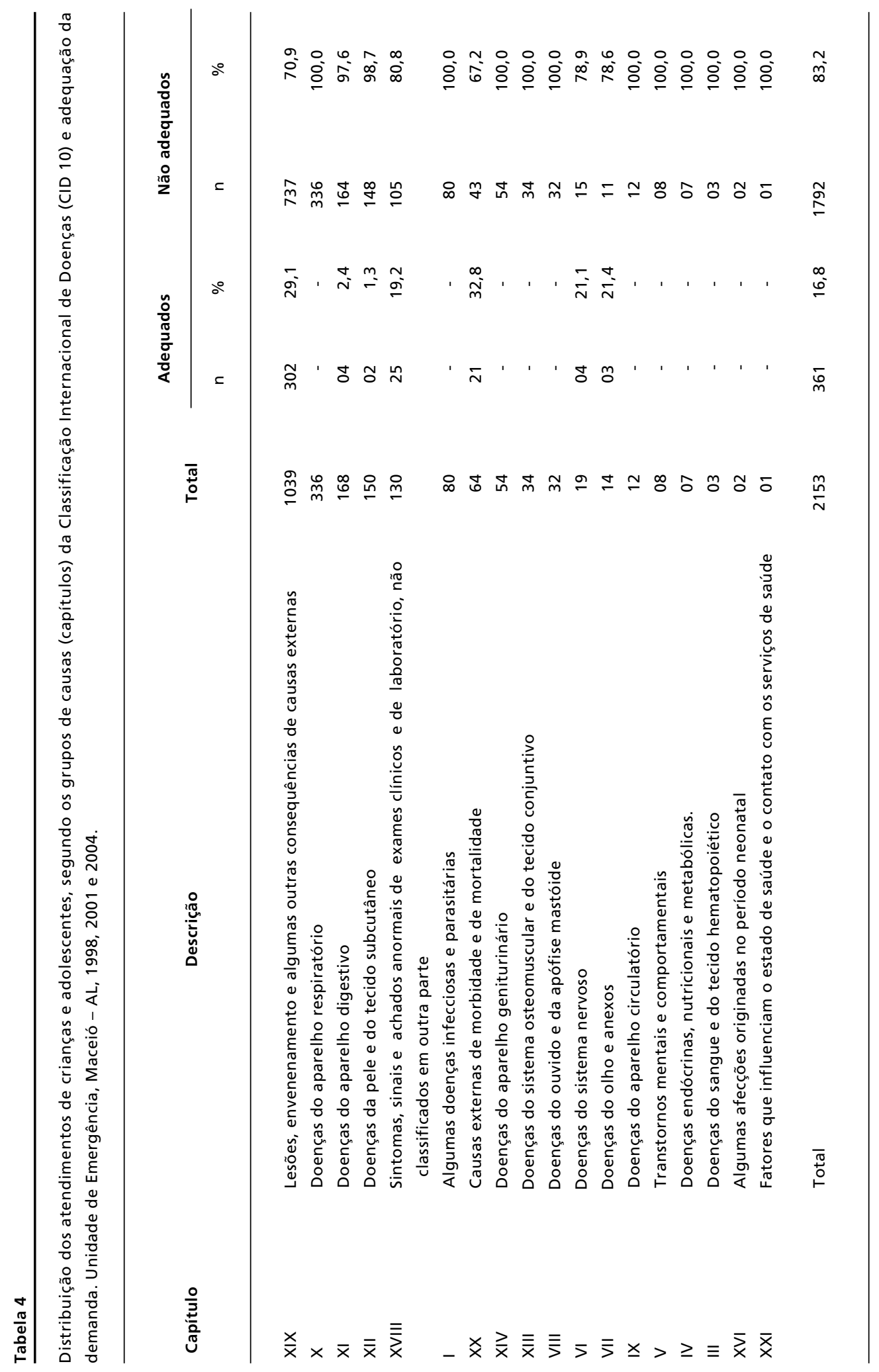




\section{Discussão}

Apenas uma pequena parcela dos atendimentos $(16,8 \%)$ na unidade de emergência correspondeu a agravos que requeriam potencial tecnológico condizente com a sua capacidade assistencial. Essa baixa adequação da demanda de atendimentos está de acordo com outros estudos que avaliaram o perfil de morbidade de usuários de serviços de emergência. 1,3,17,18

O perfil da clientela que procurou a unidade foi constituído predominantemente de usuários do sexo masculino, na faixa etária de 10 a 18 anos, atendidos à tarde, residentes em Maceió e no mesmo bairro onde se localiza a UE.

A predominância da clientela do sexo masculino é semelhante a outros estudos realizados com adolescentes, 17,18 porém chama a atenção a maior ocorrência de casos não adequados entre usuários do sexo feminino. É possível que tal fato seja devido a um maior cuidado em relação às meninas, do que aos meninos, por serem essas consideradas "frágeis" e consequentemente levadas com mais rapidez aos serviços em busca de atendimento. 19

Apesar de a faixa etária de adolescentes ter predominado neste estudo, a clínica pediátrica constituiu o setor de maior número de atendimentos, provavelmente pelo fato de atender crianças e adolescentes de 0 a 12 anos de idade.

$\mathrm{O}$ maior número de atendimentos ocorreu no período da tarde e, a maior proporção de não adequação, pela manhã, períodos esses em que as unidades básicas de saúde estão em pleno horário de atendimento e que, teoricamente, deveriam absorver grande parte dessa demanda.

Embora utilizando um critério de adequação de diagnóstico distinto, Fajardo-Ortiz e RamírezFernández ${ }^{5}$ também verificaram maior volume de atendimentos no horário diurno. Por outro lado, para esses autores, o maior percentual de não adequação ocorreu no período da tarde. Apesar dessa diferença, ressalta-se que em ambos os estudos o maior volume de casos não adequados ocorreu quando os demais serviços de saúde estavam abertos ao público.

A análise da procedência permitiu observar que a maior demanda ao serviço foi constituída de pessoas que residiam em Maceió, em bairros próximos à UE, e em municípios vizinhos, provavelmente pela acessibilidade geográfica.

Por outro lado, dificuldades de acesso a consultas na atenção básica, qualidade geral do atendimento, resolubilidade do serviço e acesso à medicação, constituem também importantes motivos que levam o usuário a procurar serviços de urgência/emergência hospitalar.2,20,21 É possível que essas tenham sido as razões para que usuários residentes em bairros distantes, onde existe ambulatórios de urgência 24 horas, tenham procurado atendimento na UE.

Quanto ao perfil de morbidade, o grupo de causas (capítulos) da CID 10 predominante foi "Lesões, envenenamentos e outras consequências de causas externas", provavelmente pela maior participação de traumatismos na amostra estudada. Esse resultado analisado conjuntamente com o predomínio da clientela de adolescentes do sexo masculino, parece estar relacionado ao crescimento do número de acidentes e da violência urbana, aos quais os meninos estão mais expostos. Resultados semelhantes foram verificados por Yamada et al. 17 e por Dubuc e Ferrari, 18 para a faixa etária de 10 a 14 anos.

Chama a atenção o alto percentual de diagnósticos não adequados $(83,2 \%)$ ocorridos no período estudado. Altos percentuais de não adequação foram também encontrados em estudos de demanda contemplando todas as faixas de idade em várias regiões do Brasil e de outros países. 1,2,3,5,22

Entre os que estudaram a população geral, a pesquisa realizada em Recife, Pernambuco, no Hospital da Restauração, encontrou 74,5\% de atendimentos não adequados. 3 Em outros cinco hospitais públicos, também em Recife, a não adequação foi $63,5 \% 2$, no Rio Grande do Sul, $60,6 \% 22$ e no México, 64,1\%.5

Cecílio ${ }^{1}$ estudou a não adequação dos diagnósticos segundo os setores em que ocorreu o atendimento, no Pronto-Socorro do Hospital Municipal de Volta Redonda, Rio de Janeiro. De acordo com este estudo, $60,0 \%$ dos atendimentos de clínica médica, $73,5 \%$ de clínica cirúrgica e $61,5 \%$ de clínica pediátrica, foram não adequados.

No presente estudo, mesmo não tendo sido utilizado internamento do usuário como critério para avaliar a adequação da demanda, chamou a atenção o alto percentual $(46,46 \%)$ de casos não adequados entre os que permaneceram internados. Esse fato pode estar relacionado a questões sociais ou à deficiência dos serviços de atenção básica para continuidade dos cuidados necessários.

O baixo percentual $(14,8 \%)$ de adequação entre usuários que não permaneceram internados pode ser devido ao fato de que, de modo geral, a atenção a alguns desses agravos - por exemplo, acidentes por escorpião, corpo estranho em orofaringe e no olho, crise convulsiva e epilepsia - não requer internamento.

Essa pesquisa contribuiu para o conhecimento do 
perfil de crianças e adolescentes atendidos na Unidade de Emergência de Maceió, ao mesmo tempo em que revelou alto percentual de não adequação da demanda àquela Unidade. Os resultados aqui apresentados corroboram achados de outros autores e sinalizam para o não atendimento pleno às necessidades de saúde dos usuários do SUS em Alagoas, seja nas unidades básicas de saúde, em ambulatório especializado ou em ambulatórios de urgência.

\section{Referências}

1. Cecílio LCO. Modelos tecno-assistenciais em saúde: da pirâmide ao círculo, uma possibilidade a ser explorada. Cad Saúde Pública. 1997; 13: 469-78.

2. Kovacs MH, Feliciano KVO, Sarinho SW, Veras AACA Acessibilidade às ações básicas entre crianças atendidas em serviço de pronto-socorro. J Pediatr (Rio J). 2005; 81: 2518 .

3. Furtado BMASM, Araújo Júnior JLC, Cavalcanti P. O perfil da emergência do Hospital da Restauração: uma análise dos possíveis impactos após a municipalização dos serviços de saúde. Rev Bras Epidemiol. 2004; 7: 279-89.

4. Vera CF, Werner AF, Massri DE. Complejidad de las consultas en un servicio de urgencia con consultorio municipal adosado. Cuad Cir. 2004; 18: 27-32.

5. Fajardo-Ortiz G, Ramírez-Fernández FA. Utilización del servicio de urgencias en un hospital de especialidades. Rev Cir Ciruj. 2000; 68: 164-8.

6. Valdés GV, Salas RP, Mohedas MC, Núnez MG, Fernández MV, Amiano FC. Evaluación de la atención urgente en un centro de salud. Adecuación de la demanda. Centro de Salud. 2002; 10: 487-494. Disponível em: http://www.medynet.com/elmedico/publicaciones/ctrosalud 2002/8/487-494.pdf

7. Jacquemot GA. Qu'est-ce qu'une urgence? Quelques reflexions sur les définitions biomédicales de l'urgence médicale. In: Bernabé J, Bonniol JL, Confiant R, L'Etang G. (Orgs.) Au Visiteur Lumineux: des îles créoles aux societés plurielles. Mélanges offerts à Jean Benoist. PetitBourg: Ibis Rouge Editions; 2000.

8. Burnet MG, Grover SA. Use of the emergency department for no urgent care during regular business hours. Can Med Assoc J. 1996; 154: 1345-51.

9. Gill JM, Riley AW. No urgent use of hospital emergency departments: urgency from the patient's perspective. J Family Pract. 1996; 42: 491-5.

10. Oterino D, Peiró S, Calvo R, Sutil P, Fernández O, Pérez G, Torre P, López MA, Sempere T. Utilización inadecuada de um servicio de urgências hospitalario. Uma evaluación com critérios explícitos. Gac Sanit. 1999; 13: 361-70.

11. Sánchez $M_{i}$ Urgências inadecuadas u oferta insuficiente? Med Clin (Barc). 2004; 123: 619-20.

Recebido em 12 de dezembro de 2008

Versão final apresentada em 16 de outubro de 2009

Aprovado em 20 de novembro de 2009

\section{Agradecimentos}

Ao Prof. Jairo Calado pela colaboração e análise estatística dos dados. À direção da Unidade de Emergência Doutor Armando Lages pelo acesso aos dados. À Fundação de Amparo à Pesquisa do Estado de Alagoas pelo suporte financeiro.
12. Andrés JAM, Nogueras RM, Castro VMTG, Bartual, VR, García PA, Pajares FG ¿Por qué los pacientes utilizan los servicios de urgencias hospitalarios por iniciativa propia? Gac Sanit. 2006; 20: 311-5.

13. Conselho Federal de Medicina. Resolução CFM $\mathrm{n}^{\circ}$ 1451/95. Disponível em: http://www.portalmedico.org.br/ resolucoes/cfm/1995/1451_1995.htm

14. Paim JS. Organização da atenção à saúde para a urgência/emergência. In: Silva LMV. (Org.) Saúde coletiva: textos didáticos. Salvador: Centro Editorial e Didático/Universidade Federal da Bahia; 1994.

15. Hinojosa CJ Ramírez ESC, Olmos JM, Villafaña JM, Jiménez JJR, Rodríguez MAP, Martín JMJ. Inadecuación de las visitas a un servicio de urgencias hospitalario y factores asociados. Aten Primaria. 2001; 28: 326-32.

16. Andrés JMA, Nogueras, RM, Bartual VR, Pajares FG, García PA. Adecuación de la demanda de atención sanitaria en servicio de urgencias hospitalarias. Med Clin (Barc). 2004; 123: 615-8.

17. Yamada ATT, Castro CGJ, Almeida MF, Garbin W, Sá ENC, Gomes MC. Estudo do perfil da demanda do serviço de pronto socorro do hospital geral de Itaquaquecetuba. São Paulo, 2002; s.n. Disponível em: http://www.bvssp.fsp.usp.br/tecom/docs/2003/yam001.pdf.

18. Dubuc IF, Ferrari RAP. Adolescentes atendidos num serviço público de urgência e emergência: perfil de morbidade e mortalidade. Rev Eletrônica Enferm. 2006; 8: 250-8.

19. The Global Fund. for children. The Dangers of Neglecting Education for Boys. 2002. Disponível em URL: http://www.globalfundforchildren.org/pdfs/gfc_dangers bo ys.pdf [2007 Dez 5]

20. Felisberto E, Leal C, Samico IC. Referência na atenção pediátrica: uma "emergência" em Pernambuco. Rev IMIP. 1994; 9: 61-9.

21. Feliciano KVO, Kovacs MH. Organização das práticas de saúde e vulnerabilidade à diarréia infantil. J Pediatr. (Rio de J.) 2000; 76: 27-36.

22. Salla J, Ghellar M, Kaufmann ML, Ilha NS, Frandoloso PR. Perfil da demanda de um serviço de Pronto Socorro, Santa Maria, RS. Saúde. 1989; 15: 71-80. 